\title{
Análise Multicritério da presença da Universidade Federal Fluminense com o uso do Método Macbeth
}

\author{
João Carlos Correia Baptista Soares de Mello \\ Departamento de Engenharia de Produção - Universidade Federal Fluminense \\ Rua Passo da Pátria, 156, São Domingos, CEP: 24210-240, Niterói, RJ, Brasil \\ jcsmello@bol.com.br \\ Eliane Gonçalves Gomes \\ Programa de Engenharia de Produção - COPPE - Universidade Federal do Rio de Janeiro \\ Caixa Postal: 68543, CEP: 21945-970, Rio de Janeiro, RJ *** \\ eggomes@pep.ufrj.br \\ Marcos Pereira Estellita Lins \\ Programa de Engenharia de Produção - COPPE - Universidade Federal do Rio de Janeiro \\ Centro de Tecnologia, Bloco F, Sala F-105, Cidade Universitária, Ilha do Fundão \\ CEP: 21945-970, Rio de Janeiro, RJ \\ lins@pep.ufrj.br \\ *** endereço para correspondências
}

Apresenta-se aqui um breve histórico da Universidade Federal Fluminense (UFF). Descreve-se o seu processo de interiorização e as diversas atividades realizadas nos municípios com presença desta instituição. Alguns conceitos do Apoio Multicritério à Decisão são apresentados e seguidamente usados para uma avaliação da interiorização no que diz respeito ao impacto em cada município. O método MACBETH é descrito e usado para a análise da presença e/ou visibilidade da UFF nestes municípios.

Palavras-chave: Multicritério; Interiorização universitária; MACBETH.

This paper presents a brief history of Universidade Federal Fluminense (UFF), and describes his expansion to some country cities. Afterwards multicriteria basic concepts are presented and used to evaluate UFF's presence in each municipal district. The multicriteria method choose is the MACBETH approach.

Keywords: Multicriteria Decision Aid; Universitary presence; MACBETH.

\section{INTRODUÇÃO}

O planejamento estratégico, importante para qualquer instituição, necessita inicialmente de conhecimento interno, ou seja, conhecimento das características e das formas de atuação da instituição. A Universidade Federal Fluminense (UFF) vem, desde algumas décadas, expandindo suas atividades principalmente no Estado do Rio de Janeiro, sem contudo realizar uma avaliação metódica desta expansão. No máximo são emitidas opiniões de pessoas envolvidas neste processo, ou de seus críticos.

Este artigo tem como primeiro objetivo quantificar um parâmetro necessário à análise sistemática desta expansão: o impacto das atividades da UFF em cada município. Como são realizadas atividades de vários tipos (ensino de nível superior, de nível médio, de extensão, etc.) e há várias formas de medi-las, é necessário recorrer a um método que permita levar em conta todos esses aspectos. Por outro lado, a avaliação de cada atividade em si é, por natureza, essencialmente qualitativa. Como se pretende que a avaliação final seja quantitativa, é mister dispor de um método que, além de lidar com vários critérios, permita transformar escalas ordinais em cardinais. Para tal, é utilizado o método MACBETH (Bana e Costa \& Vansnick, 1994, 1997) que integra os métodos multicritério de apoio à decisão.

Na seção seguinte é apresentado um breve histórico do processo de interiorização das atividades da UFF, seguida de alguns aspectos relacionados ao Apoio Multicritério à Decisão, com especial ênfase ao método MACBETH. Uma revisão deste método é o segundo 
objetivo deste artigo. A Seção 4 traz os critérios adotados na análise, seguida dos julgamentos de valor dos decisores. Os resultados obtidos, as conclusões $e$ as referências bibliográficas utilizadas são apresentados, respectivamente, nas seções 6,7 e 8 .

\section{BREVE HISTÓRICO DA UNIVERSIDADE FEDERAL FLUMINENSE E DE SUA INTERIORIZAÇÃO}

A Universidade Federal Fluminense (UFF) foi criada na década de 60 a partir da fusão de várias faculdades isoladas do município de Niterói, RJ, onde é sua sede. Posteriormente foram agregados uma Escola de Engenharia em Volta Redonda (inicialmente com um curso de Engenharia Metalúrgica e, a partir de 2001, também de Engenharia de Produção e Engenharia Mecânica), uma escola de Serviço Social em Campos dos Goytacazes, e dois colégios agrícolas em Pinheiral $e$ Bom Jesus do Itabapoana (com cursos técnicos em Agropecuária) (COSEAC, 2001).

A partir de 1984, através de um convênio com a Prefeitura de Santo Antônio de Pádua, a UFF começou um projeto de interiorização de cursos de graduação, tendo estabelecido um curso de licenciatura em Matemática nesta cidade. Este curso funciona até hoje em instalações cedidas, embora já possua um terreno para construção das futuras instalações. Enquanto que a responsabilidade do corpo docente é exclusiva da UFF, a prefeitura contribui com facilidades para deslocamento e alojamento dos professores não residentes na região.

$\mathrm{Na}$ década de 90, a interiorização se expandiu para os municípios de Angra dos Reis (curso de Pedagogia), Cabo Frio (Ciências Contábeis), Itaperuna (Administração), Macaé (Administração e Ciências Contábeis) e Miracema (Ciências Contábeis). Nestas cidades as instalações são cedidas e o corpo docente, embora da UFF, conta com suporte financeiro da prefeitura. Assim, o governo municipal participa das decisões sobre abertura de vagas no vestibular.

A UFF conta ainda com duas fazendas escola em Cachoeiras de Macacu e Iguaba Grande, com pouca atividade. Possui também um campus avançado em Oriximiná (PA), onde há campo para estágio de alunos de áreas sociais e de saúde, que atuam no atendimento à população local. A Figura 1 mostra a distribuição espacial da presença da UFF no país.

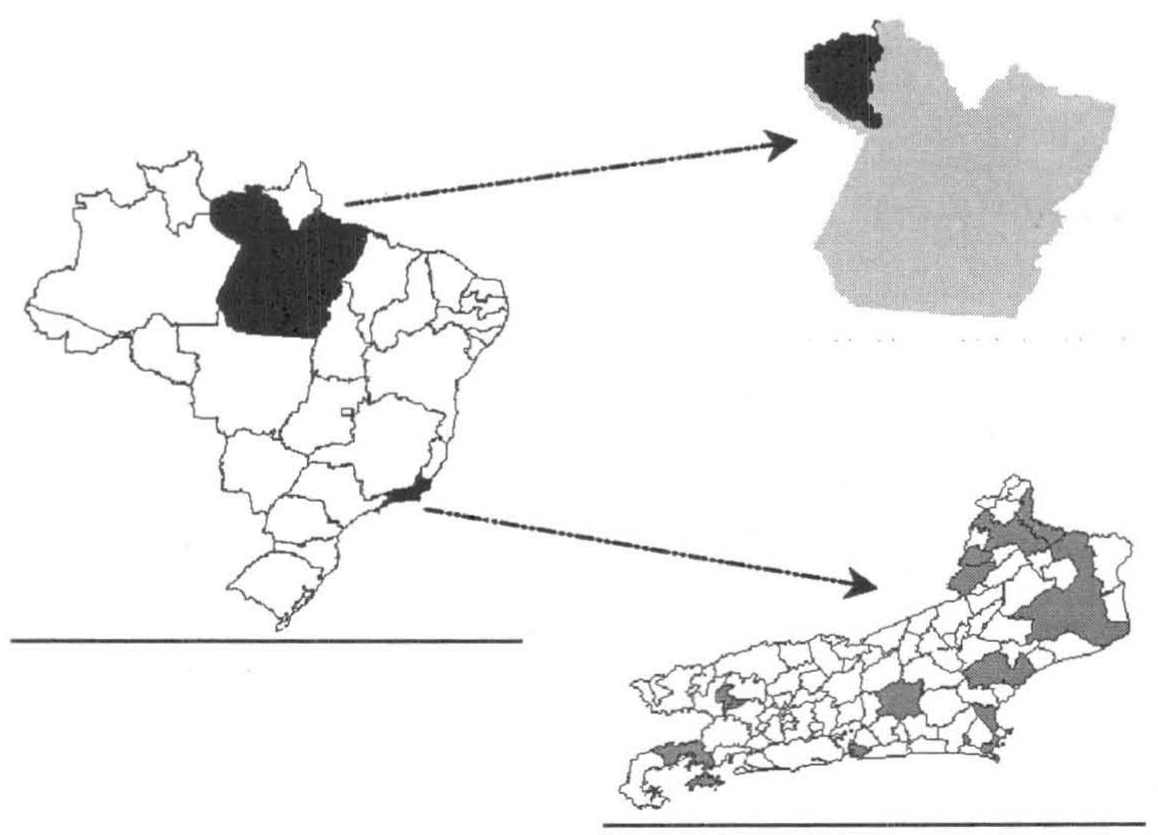

Figura 1: Distribuição espacial da presença da UFF nos municípios dos estados do Rio de Janeiro e Pará. 


\section{Apoio Multicritério à Decisão}

O Apoio Multicritério à Decisão pode ser definido como um conjunto de técnicas de apoio à tomada de decisão, que têm a finalidade de investigar um número de alternativas, considerando múltiplos critérios e objetivos em conflito. É possível gerar soluções de compromisso $e$ uma hierarquização das alternativas, de acordo com o grau de atração destas para o tomador de decisão (Gomes, 2002).

Segundo Bouyssou (1993), uma abordagem multicritério apresenta as seguintes vantagens:

- Construção de uma base para o diálogo entre os intervenientes utilizando diversos pontos de vista comuns;

- Maior facilidade em incorporar incertezas aos dados sobre cada ponto de vista;

- Interpretar cada alternativa como um compromisso entre objetivos em conflito.

Este último argumento destaca o fato de que raramente será encontrada uma situação em que exista uma alternativa superior às restantes sobre todos os pontos de vista.

Em um problema multicritério é necessário, em primeiro lugar, estabelecer claramente qual o objetivo da análise. Classicamente podem ser definidas três problemáticas multicritério: ordenação, escolha e alocação em classes (Barba-Romero e Pomerol, 1997). Este artigo lida com a problemática de ordenação.

Deve-se ainda definir quem atua como decisor, ou seja, aquele que emite juízos de valor sobre as alternativas $e$ os critérios (neste artigo, um dos autores), as alternativas (os municípios onde a UFF está presente), $e$ o método a ser usado.

Este artigo faz a quantificação da presença da UFF através de um critério único de síntese e, por isso, usa métodos da chamada Escola Americana (Barba-Romero e Pomerol, 1997). Os métodos de soma ponderada são os mais comumente utilizados. O problema maior destes métodos é a determinação do vetor de pesos que melhor retrate a opinião do decisor sobre a influência de cada critério na construção do critério síntese. Os métodos de tradeoff (Barba-Romero e Pomerol, 1997), AHP (Saaty, 1980) e MACBETH (Bana e Costa \& Vansnick, 1994) são alguns dos usados para resolver este problema. A escolha do método MACBETH deve-se a dois fatores principais, a saber:

- Permite a transformação de avaliações qualitativas em quantitativas, o que também é feito pelo método $A H P$. Todos os critérios deste artigo têm avaliação qualitativa.

- Não permite nenhum grau de inconsistência nos julgamentos do decisor, estabelecendo um processo interativo de revisão dos julgamentos, inclusive sugerindo quais os que devem ser revistos. Ressalta-se que o método AHP não apresenta esta possibilidade, arbitrando um valor de $10 \%$ para a inconsistência máxima nos julgamentos.

Apresenta-se a seguir uma revisão sobre o método MACBETH.

\subsection{Método MACBETH}

\subsubsection{Aspectos gerais}

Na resolução do presente problema multicritério existem duas questões essenciais, a saber:

- Para cada critério, determinar uma escala de valores, ou seja, atribuir notas a cada alternativa. Em alguns casos existe uma forma natural de fazer essa atribuição, sendo custo de uma mercadoria o exemplo clássico. Em outros casos a avaliação é qualitativa, sendo necessário transforma-la em quantitativa.

- Tendo as "notas" de cada alternativa relativas a cada critério, é necessário agrega-las em uma nota única através de uma soma ponderada. O problema consiste na atribuição de pesos aos vários critérios, respeitando as opiniões dos decisores.

Estes dois problemas podem ser resolvidos com o auxílio do método MACBETH (Measuring $\underline{\text { Attractiveness by a }}$

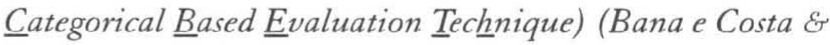
Vansnick, 1994). Para o primeiro deles é usado o módulo "scores" do programa MACBETH. Este módulo permite atribuir notas a cada alternativa através de uma comparação pareada. Dadas duas alternativas, o decisor deve dizer qual a mais atrativa (tem maior nota) e qual o grau desta atratividade em uma escala semântica que tem correspondência com uma escala ordinal. $O$ próprio programa faz a análise de coerência cardinal (transitividade) e semântica (relações entre as diferenças), sugerindo, em caso de incoerência, como 
resolvê-la. Por programação linear são sugeridos uma escala de notas e os intervalos em que elas podem variar sem tornar o problema inconsistente (PPL inviável). $\quad E$ ainda facultado ao decisor ajustar graficamente o valor das notas atribuídas, dentro dos intervalos permitidos. Segundo Bana e Costa E Vansnick (1997) somente após este ajuste, com a introdução dos conhecimentos dos especialistas, é que fica caracterizada a construção da escala cardinal de valores.

Para o segundo problema apontado (atribuição de pesos e construção da função que conduz ao critério síntese), utilizou-se o módulo "weights" do programa MACBETH, que atua de maneira semelhante ao módulo "scores". Ao contrário do método AHP que compara a importância dos critérios diretamente, o MACBETH faz a comparação de forma indireta, considerando alternativas fictícias que representam cada um dos critérios. A alternativa fictícia a representa o critério $j$ quando apresenta a melhor nota em $j$ e a pior em todos os outros critérios. $E$ ainda introduzida uma outra alternativa, correspondente a um critério artificial, com a pior nota em todos os critérios, com a finalidade de evitar que um critério real tenha peso nulo. $A$ eventual atribuição de peso zero a um critério relevante violaria o axioma da exaustão (Roy e Bouyssou, 1993). Através da comparação da atratividade das alternativas são atribuídos os pesos aos critérios de forma análoga ao procedimento realizado no módulo "scores".

A principal diferença entre os dois módulos é que, enquanto no "scores" há a restrição de as notas ocuparem todo o intervalo definido, no "weights" o peso menor ocupa o valor mais baixo da escala, mas em vez de fixar o valor do maior peso obriga a que a soma de todos os pesos seja igual à unidade.

A seguir, formalizam-se os aspectos gerais aqui apresentados.

\subsubsection{Aspectos teóricos}

\section{Diferença de Atratividade}

No método MACBETH, quando ao decisor forem solicitados julgamentos de valor sobre as ações potenciais (alternativas) em uma determinada situação, ele o fará em termos da atratividade que sente por esta alternativa. Esta tarefa é definida (Bana e Costa E Vansnick, 1995) como a construção de uma função-critério $v$, tal que:
- para $a, b \in A, v(a)>v(b)$ se e somente se para o avaliador a é mais atrativa (localmente) que b (a $P$ b);

- qualquer diferença positiva $v(a)>v(b)$ representa numericamente a diferença de valor entre $a$ e $b$, com a $P$ $b$ sempre em termos de um ponto de vista fundamental $j$ $(P V F)$, ou critério $j$.

Assim, para $a, b, c, d \in A$ com a mais atrativa que $b$ e $c$ mais atrativa que $d$, se verifica que $v(a)-v(b)>v(c)$ $v(d)$ se e somente se " $a$ diferença de atratividade entre a $e$ $b$ é maior que a diferença de atratividade entre $c e d$ ".

A questão fundamental neste método é (Bana e Costa $\mathcal{E}$ Vansnick, 1995): "Dados os impactos $i_{j}(a)$ e $i_{j}(b)$ de duas ações potenciais a e $b$ de $A$ segundo um ponto de vista fundamental $P V F$, sendo a julgada mais atrativa que $b, a$ diferença de atratividade entre a e b é "fraca", "forte", ...?"

É introduzida uma escala semântica formada por categorias de diferença de atratividade, com o objetivo de facilitar a interação entre o decisor e o analista. $O$ decisor deverá escolher uma, e somente uma, entre as categorias apresentadas.

Se por um lado, o método MACBETH introduz um intervalo da reta real associado a cada uma das categorias, por outro lado, este intervalo não é fixado a priori, sendo determinado simultaneamente com a escala numérica de valor $\mathbf{v}$ que está sendo procurada.

Assim, este método liga-se ao problema teórico de representação numérica de semi-ordens múltiplas por limiares constantes de Doignon (1987), representado por $m$ relações binárias $\left(P^{(1)}, P^{(2)}, \ldots, P^{(k)}, \ldots, P^{(m)}\right)$, onde $P^{(k)}$ representa a relação de preferência tanto mais forte quanto maior é $k$, dado um critério $j$.

As preferências são representadas por uma função $v$ e por funções limiares $s_{k}: a P^{(k)} b, s_{k}<v(a)-v(b)<s_{k+1}$, ou seja, é possível representar numericamente categorias semânticas de diferença de atratividade através de um intervalo de números reais.

Não há restrição ao número de categorias semânticas a ser utilizado. No entanto, uma pessoa é capaz de avaliar, simultaneamente, um número limitado de classes quando da expressão de um juízo absoluto de valor, sendo algo em torno de sete fatores.

No MACBETH, a expressão dos julgamentos do decisor é feita por uma escala semântica formada por 
seis categorias, de dimensão não necessariamente igual:

- $C_{1}$ diferença de atratividade muito fraca $\rightarrow C_{1}=\left[s_{1}\right.$ $\left.s_{2}\right]$ e $s_{1}=0$

- $C_{2}$ diferença de atratividade fraca $\left.\left.\rightarrow C_{2}=\right] s_{2}, s_{3}\right]$

- $\quad C_{3}$ diferença de atratividade moderada $\rightarrow C_{3}=J s_{3^{3}} s_{4} l$

- $C_{4}$ diferença de atratividade forte $\left.\rightarrow C_{4}=\right] s_{4}, s_{5} l$

- $\quad C_{5}$ diferença de atratividade muito forte $\left.\left.\rightarrow C_{5}=\right] s_{5}, s_{6}\right]$

- $C_{6}$ diferença de atratividade extrema $\left.\rightarrow C_{6}=\right] s_{6^{\prime}}+[$

As categorias são delimitadas por limiares constantes $s_{1}$, ..., $s_{6}$, determinados simultaneamente à obtenção da escala de valor $v$.

A Figura 2 (Corrêa, 1996) ilustra um exemplo de problema de semi-ordens.

O decisor fez seus julgamentos absolutos de diferença de atratividade entre as três alternativas presentes, obtendose os limiares e os valores para as alternativas, hierarquizando a, b e c com 100, 93 e 30 pontos, respectivamente.

$O$ resultado indica que a diferença de valor entre as alternativas a e $b$ é sete unidades $(v(a)-v(b)=7)$, o que está de acordo com os limiares da categoria $C_{1}$ (diferença de atratividade muito fraca), definida entre os valores $0 e$ 10 unidades (para este exemplo). A diferença de atratividade entre as alternativas b e c foi considerada como sendo forte pelos decisores, ou seja, categoria $C_{4} \cdot A$ escala construída gerou uma diferença de valor entre as alternativas de 63 unidades $(v(b)-v(c)=63)$, valor este que está entre os limites da categoria $C_{4}, 44$ e 67 unidades. Para o par $(a, c)$, a diferença de atratividade foi considerada forte (categoria $C_{5}$ ), devendo a diferença de valor entre as duas alternativas estar entre os limiares da categoria $C_{5}$, ou seja, entre 67 e 92 unidades. A diferença é 70 pontos $(v(a)-v(c)=70)$, dentro do esperado.

\section{Matriz de juízos de valor}

Bana e Costa \& Vansnick (1995) propõem a construção de matrizes de juízos de valor para facilitar a expressão dos julgamentos absolutos de diferença de atratividade entre os pares de alternativas. A Figura 3 mostra a matriz triangular superior construída para cada critério, na qual se supõe que $A=\left\{a_{n^{\prime}} a_{n-1}, \ldots, a_{1}\right\}$ é o conjunto de $n$ alternativas a avaliar, e que estas estão ordenadas por ordem decrescente de atratividade $a_{n} P a_{n-1} P \ldots P a_{P}$ não existindo indiferença em nenhum caso para este critério.

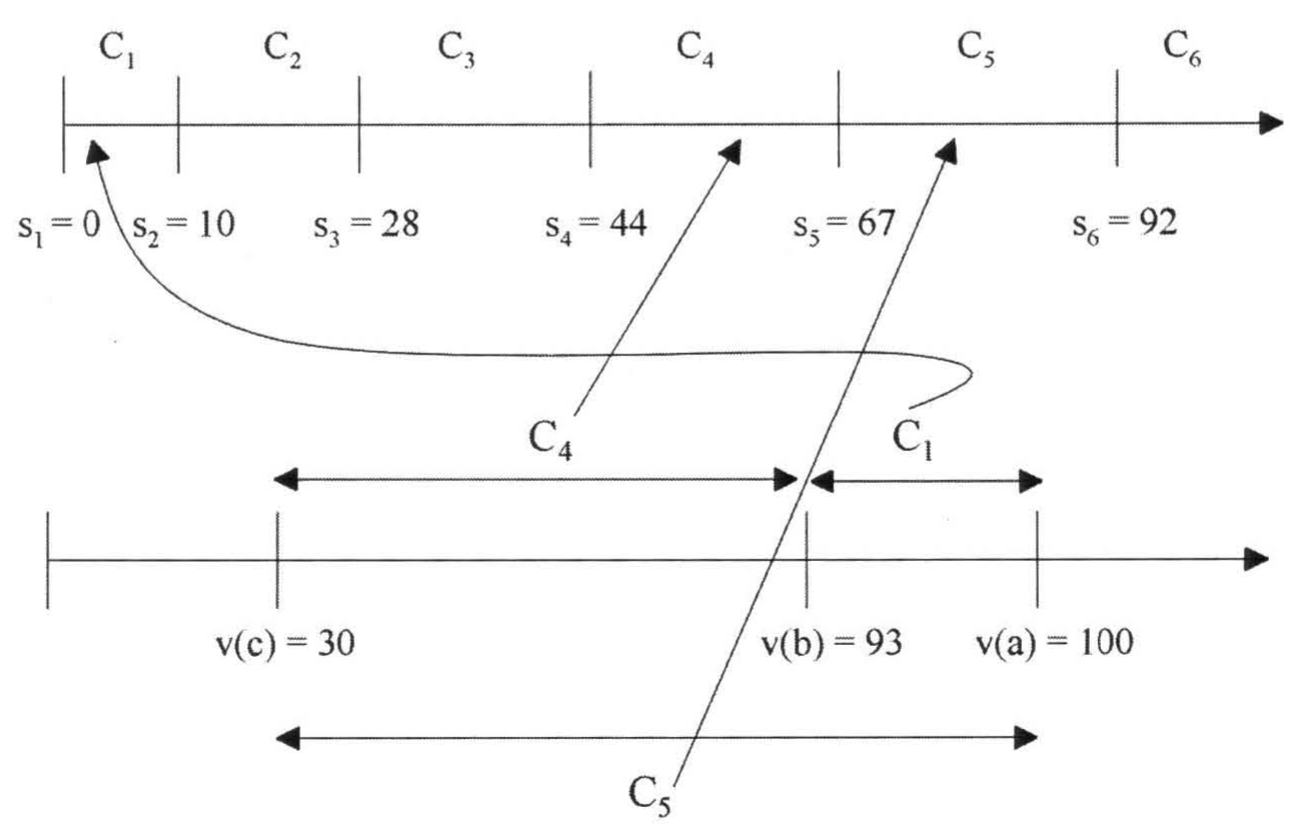

Figura 2: Exemplo de representação numérica de semi-ordens múltiplas por limiares constantes de Doignon. 


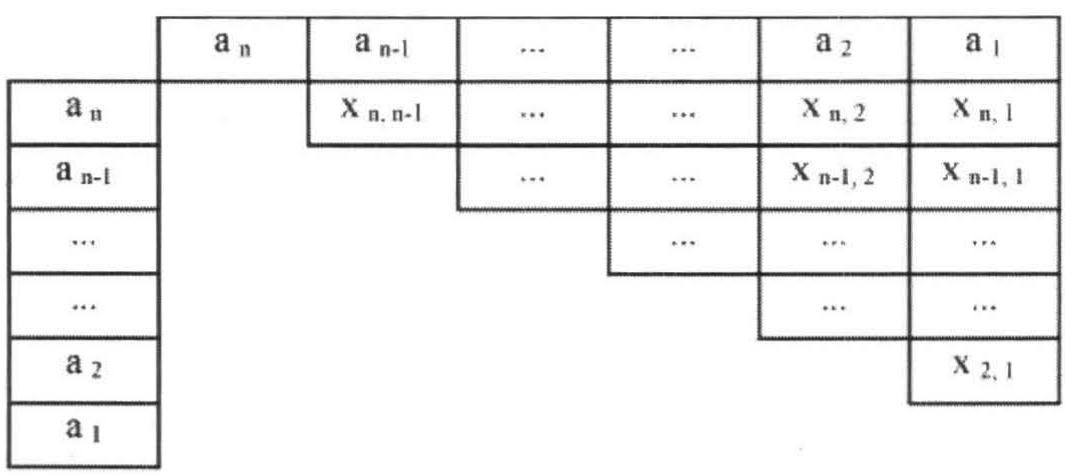

Figura 3: Matriz de juízos de valor para avaliação local das ações.

Cada elemento $x_{i, j}$ da matriz toma o valor $k(k=1,2,3$, $4,5,6)$ se o decisor julgar que a diferença de atratividade do par $(a, a)$ pertence à categoria $C_{k}$. Estes números não têm significado matemático; servem apenas como indicadores semânticos de qual categoria de diferença de atratividade foi atribuída ao par respectivo.

\section{Inconsistência nos Julgamentos de Valor}

Nos casos em que as matrizes de valor são grandes, a avaliação de todas as alternativas de maneira coerente torna-se difícil. Nestes casos, é comum o aparecimento de inconsistências nos julgamentos de valor do decisor. Há dois tipos de inconsistências: semântica (quando a atribuição de categoria de diferença de atratividade a um par de alternativas não é logicamente aceitável) $e$ cardinal (se a representação dos julgamentos não é possivel através de uma escala cardinal dentro dos números reais).

Inconsistência semântica

Suponha que um decisor atribuiu aos pares de alternativas $(a, b)$ e $(b, c)$ categorias de diferença de atratividade $C_{k}$ e $C_{k}$, respectivamente. Sendo $k>k$, então a é mais atrativa que $b$ de forma mais intensa do que bé mais atrativa que c. A transitividade exige que a diferença de atratividade entre a e c pertença a uma

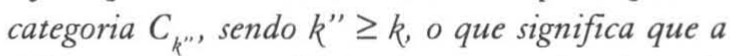
diferença de atratividade entre o par $(a, c)$ é pelo menos tão grande quanto aquela entre o par $(a, b)$.

A utilização do teste de consistência em casos reais faz

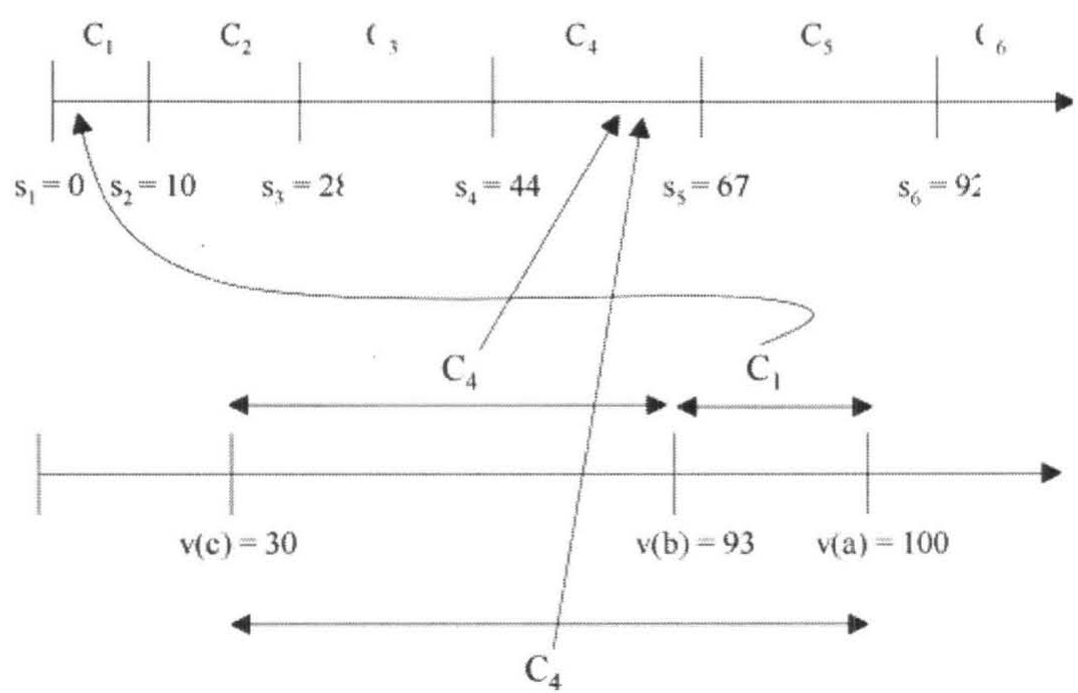

Figura 4: Exemplo de inconsistência cardinal. 
com que os decisores refaçam seus juízos de valor quando envolvidos em alguma situação de inconsistência.

\section{Inconsistência cardinal}

A inconsistência cardinal ocorre em situações em que o decisor gera um conjunto de julgamentos que são semanticamente consistentes, mas que não podem ser representados numericamente. É conhecido da teoria (Doignon, 1987; Bana e Costa \& Vansnick, 1995) que a representação numérica de semi-ordens múltiplas por limiares constantes nem sempre é possivel.

A Figura 4 (Corrêa, 1996) representa uma situação com inconsistência cardinal nos julgamentos de valor do decisor.

O julgamento da diferença de atratividade entre as alternativas a e c foi indicada pelo decisor como pertencente à categoria $C_{4} \quad A$ diferença de valor entre as alternativas deve ser um valor numérico entre $s_{4}$ e $s_{5}$. Pela Figura 4 verifica-se que isto não é possível de ser obtido, uma vez que a diferença de valor entre a e c,v(a)$v(c)=70$ é maior do que o limiar $s_{5}=67$, tornando-se impossível construir os limiares constantes

(representados na reta superior), pois a condição teórica não pode ser respeitada, e o problema não tem solução, apesar de ser semanticamente consistente.

Cabe destacar que o que se deseja é que a diferença de valor entre as alternativas seja um número compreendido entre os valores absolutos $s_{k}$ e $s_{k+1}$. Uma vez que a diferença de atratividade entre duas alternativas é, por exemplo, forte, isto não significa que a amplitude da categoria $C_{4}$ é grande, mas sim que os valores absolutos dos limiares desta categoria são elevados.

\section{Formulação Matemática}

Matematicamente, o método MACBETH é constituído por quatro problemas de programação linear (PPLs) seqüenciais que realizam a análise de consistência cardinal, a construção da escala de valor cardinal $e$ revelam fontes de inconsistência.

\section{$\underline{1}^{\circ} \underline{\mathrm{PPL}}$}

$O 1^{\circ} P P L$ verifica a existência de inconsistências cardinais. Matematicamente é representado por (I):
$\operatorname{Min} c$

sujeito a

r0) $s_{p}, \ldots, s_{6} \geq 0 ; v(a) \geq 0, \forall a \in A ; c \geq 0$

r1) $s_{1}=0$

r2) $v\left(a_{1}\right)=0$, onde $\forall a \in A$, vale $a P a_{1}$

r3) $k=\{2, \ldots, 6\}: s_{k}-s_{k-1} \geq 1000$

r4) $k=\{1, \ldots, 6\},(a, b) \in C_{k}: v(a)-v(b) \geq s_{k}+1-c$

r5) $k=\{1, \ldots, 5\},(a, b) \in C_{k}: v(a)-v(b) \leq s_{k+1}-1+c$

A Função Objetivo (FO) de (I) é a minimização da variável auxiliar c, cuja utilidade é verificar se há inconsistência nos julgamentos do decisor (para $c=0$, não há inconsistências).

A restrição r0 garante a condição de não-negatividade para todas as variáveis do problema. As restrições rle $r 2$ fixam uma origem para a escala, garantindo que o limiar inferior da categoria de diferença de atratividade $C_{1}$ e o valor da alternativa menos atrativa sejam iguais a zero. $O$ conjunto de restrições $r 3$ estabelece que o tamanho mínimo de cada categoria seja igual a 1000 unidades, valor arbitrário escolhido de maneira a que o erro introduzido nas duas restrições seguintes não tenha um valor significativo.

As restrições $r 4$ e $r 5$ são a aplicação da fórmula de Doignon para o problema de semi-ordens múltiplas: a $P^{(k)} b, s_{k}<v(a)-v(b)<s_{k+1}$, ou seja, para cada par de alternativas deve-se garantir que a diferença de valor esteja entre os limites da categoria de diferença de atratividade que lhes foi atribuída. De maneira a ser possivel a utilização de programação linear, a equação acima foi transformada em duas, representadas pelas restrições $r 4$ e $r 5$. Como em programação linear não é possível a utilização de desigualdades estritas foi incluída uma constante, com o valor de 1 unidade, fazendo com que a condição teórica seja respeitada.

Quando há inconsistências cardinais o problema de representação numérica de semi-ordens múltiplas não tem solução. Com a introdução da variável c, o PPL (I) tem sempre solução, ou seja, sempre irá produzir uma escala que represente os julgamentos de valor do decisor. Quando o valor da FO for diferente de zero ( $c \neq$ 0) há inconsistências, ou seja, a escala não representa 
fielmente os julgamentos do decisor.

$\underline{2}^{\circ} \underline{\mathrm{PPL}}$

O $2^{\circ}$ PPL é responsável pela construção da escala de valor cardinal que representa o conjunto de julgamentos do decisor. É representado pelo PPL (II).

$\operatorname{Min}\{\Sigma[\varepsilon(a, b)+\eta(a, b)]+\Sigma[\alpha(a, b)+\delta(a, b)]\}$

r0) $s_{p}, \ldots, s_{6} \geq 0 ; v(a) \geq 0, \forall a \in A ; c \geq 0$

r1) $s_{1}=0$

r2) $v\left(a_{1}\right)=0$, onde $\forall a \in A$, vale $a a_{1}$

r3) $k=\{2, \ldots, 6\}: s_{k}-s_{k-1} \geq 1000$

r4) $k=\{1, \ldots, 6\},(a, b) \in C_{k}: v(a)-v(b) \geq_{s_{k}}+1$

r5) $k=\{1, \ldots, 5\},(a, b) \in C_{k}: v(a)-v(b) \leq s_{k+1}-1$

rb) $k=\{1, \ldots, 5\},(a, b) \in C_{k}: v(a)-v(b)=0.5\left(s_{k}+s_{k+1}\right)+\varepsilon(a$, b) $-\eta(a, b)$

r7) $(a, b) \in C_{6} \cdot v(a)-v(b)=s_{6}+1-\alpha(a, b)+\delta(a, b)$

O problema da representação numérica de semi-ordens por limiares constantes, quando construído conforme o método MACBETH, admite infinitas soluções. $O$ critério adotado por Bana e Costa \& Vansnick (1995) para a escolha da solução é a minimização dos desvios absolutos entre a diferença de valor de duas alternativas $(v(a)-v(b))$ e o ponto médio da categoria de diferença de atratividade à qual pertencem $\left(0,5^{*}\left(s_{k+1}+s_{k}\right)\right)$, para $k \neq 6$. Para a categoria $C_{6}$ o critério escolhido foi a minimização dos desvios absolutos entre a diferença de valor das alternativas e o ponto $s_{6}+1$. A FO de (II) é, portanto, a minimização da soma dos desvios absolutos.

$A$ restrição $r 0$ garante a condição de não-negatividade para todas as variáveis do problema. As restrições $r 1, r 2 e$ $r 3$ são iguais às do PPL (I). As restrições $r 4$ e $r 5$, da mesma forma, são similares àquelas já apresentadas, apenas não havendo necessidade de incluir a variável auxiliar c, uma vez que todas as fontes de inconsistência já foram analisadas.

A restrição r6 faz com que a diferença de valor entre o par $(a, b)$ seja igual ao valor central da categoria de diferença de atratividade à qual pertencem, acrescido de um desvio absoluto. Esta restrição é aplicada a todos os pares dealternativas pertencentes $a C_{k}$ com $k=1, \ldots, 5$. Para os pares que possuem diferença de atratividade extrema, ou seja, $k=6$, a restrição $r 7$ faz com que a diferença de valor entre o par de alternativas seja igual ao limiar inferior da categoria mais 1 unidade, mais o desvio absoluto. Ou seja, procura fazer com a diferença de valor entre pares de alternativas que pertençam a categoria $C_{6}$ esteja o mais próximo possível do limiar inferior desta categoria.

\section{$\underline{3} \underline{0} \underline{\mathrm{e}} \underline{4}^{\circ} \underline{\text { Problemas }}$}

Quando no PPL (I) c é diferente de zero, há inconsistências nos julgamentos de valor do decisor. $O$ procedimento mais adequado é uma revisão dos juízos inicias, discutindo com decisor possíveis modificações para tentar ultrapassar problemas de inconsistência. Os PPLs (III) e (IV) evidenciam as possiveis causas de inconsistência. Apresentam a mesma FO, diferindo apenas nas restrições.

$\operatorname{Min}\{\Sigma[\alpha(a, b)+\beta(a, b)]\}$

r0) $s_{1}, \ldots, s_{6} \geq 0 ; v(a) \geq 0, \forall a \in A ; c \geq 0$

r1) $s_{1}=0$

r2) $v\left(a_{1}\right)=0$, onde $\forall a \in A$, vale a $P a_{l}$

r3) $k=\{2, \ldots, 6\}: s_{k}-s_{k-1} \geq 1000$

r4) $k=\{1, \ldots, 6\},(a, b) \in C_{k}: v(a)-v(b) \geq s_{k}+1$

r5) $k=\{1, \ldots, 5\},(a, b) \in C_{k}: v(a)-v(b) \leq s_{k+1}-1$

r6) $k=\{1, \ldots, 6\},(a, b) \in C_{k}: v(a)-v(b)=s_{k}+1-\alpha(a$,

b) $+\delta(a, b)$

$r 7) k=\{1, \ldots, 5\},(a, b) \in C_{k}: v(a)-v(b)=s_{k+1}-1+\beta(a$, b) $-\gamma(a, b)$

$\operatorname{Min}\{\Sigma[\alpha(a, b)+\beta(a, b)]\}$

r0) $s_{p}, \ldots, s_{6} \geq 0 ; v(a) \geq 0, \forall a \in A ; c \geq 0$

r1) $s_{1}=0$

r2) $v\left(a_{1}\right)=0$, onde $\forall a \in A$, vale a $P a_{1}$ 
r3) $k=\{2, \ldots, 6\}: s_{k}-s_{k-1} \geq 1000$

r6) $k=\{1, \ldots, 6\},(a, b) \in C_{k}: v(a)-v(b)=s_{k}+1-\alpha(a$, b) $+\delta(a, b)$

$r 7) k=\{1, \ldots, 5\},(a, b) \in C_{k}: v(a)-v(b)=s_{k+1}-1+\beta(a$, b) $-\gamma(a, b)$

A FO minimiza a soma das variáveis $\alpha(a, b)$ e $\beta(a, b)$, fazendo ressaltar em (III) e em (IV) pares de alternativas cuja identificação com as categorias respectivas especificadas pelo decisor introduzem problemas de inconsistência. Ou seja, aqueles para os quais os valores de $\alpha(a, b)$ ou de $\beta(a, b)$ são diferentes de zero na solução ótima de (III) ou. Como resultado, é sugerida ao decisor uma matriz alterada que leve à consistência.

A diferença entre as soluções ótimas destes dois problemas está no fato de restringirem (III) ou não (IV) as soluções possiveis a valores das variáveis $\alpha(a, b)$ e $\beta(a$, b) não superiores ao valor de c, pela introdução (III) ou não (IV) das restrições $r 4$ e $r$.

\section{Determinação de pesos para os critérios}

De posse dos julgamentos absolutos de valor segundo cada um dos critérios, é necessário a obtenção de informações de natureza inter-critérios (representadas por constantes de escala, taxas de substituição ou pesos), para uma avaliação global das alternativas. No método $M A C B E T H$, cada critério é representado por uma alternativa fictícia que tenha a melhor avaliação possível nesse critério e a pior nos demais critérios.

Para que não sejam perdidas informações a respeito do critério considerado menos atrativo, deve-se introduzir na matriz de juízos de valor uma alternativa fictícia extra, que deve possuir o pior nível de impacto em todos os pontos de vista fundamentais. A inclusão desta alternativa evita que seja atribuído peso nulo a algum critério, o que violaria o axioma da exaustão de Roy (Roy e Bouyssou, 1993).

Com este conjunto de julgamentos, o método MACBETH é executado primeiramente para a verificação de eventuais inconsistências semânticas e cardinais e, posteriormente, para a determinação de uma escala de valor cardinal que represente os julgamentos de valor do decisor. Os PPLs são semelhantes aos anteriores, excetuando-se a restrição de normalização adicionada neste módulo.

\section{CRITÉRIOS ADOTADOS NO ESTUDO DE CASO}

Com o objetivo de analisar a intensidade da atuação da UFF nos municípios onde há alguma presença desta instituição, foram selecionados fatores que formam uma família coerente de critérios, no sentido em que descrevem o problema o melhor possível, são coerentes $e$ não redundantes.

Os critérios adotados foram:

- Tipo de curso: neste critério avalia-se se existe algum curso no município analisado, se é de nível superior ou médio e quantos cursos existem. Não são feitas considerações subjetivas sobre interesse ou qualidade dos cursos.

- Regularidade do acesso: aqui é verificado se nos municípios onde há cursos, o ingresso é feito de forma regular, ou seja, se em todos os anos há ingresso nos dois semestres. Este critério fornece uma indicação da estabilidade do curso, ou se este apresenta problemas de funcionamento. Apresenta certa redundância com o anterior, visto que um município que não tenha cursos também não apresentará regularidade do acesso. Entretanto, se este critério não fosse considerado, o problema não estaria representado de forma completa.

- Atuação junto à comunidade: visa identificar outras formas de atuação da instituição além dos cursos oferecidos, tais como, atendimento hospitalar, serviços culturais (cinema, teatro, orquestra sinfônica), atendimento social, cursos de extensão, convênios com empresas. Este critério pode ser interpretado como uma das formas principais de visibilidade da instituição perante a comunidade, principalmente para as pessoas de menor instrução.

- Instalações: é avaliado se as atividades são realizadas em sede própria ou em imóveis cedidos elou compartilhados. Em locais onde a sede é própria, verifica-se se as instalações atendem plenamente às necessidades da instituição. Onde não há sede própria, a análise fica sem sentido, pois tudo que existe pode ser considerado provisório.

- Corpo docente: neste critério considera-se a existência de professores lotados especificamente e de forma permanente para atividades no município. Devese considerar ainda se os professores lotados no 


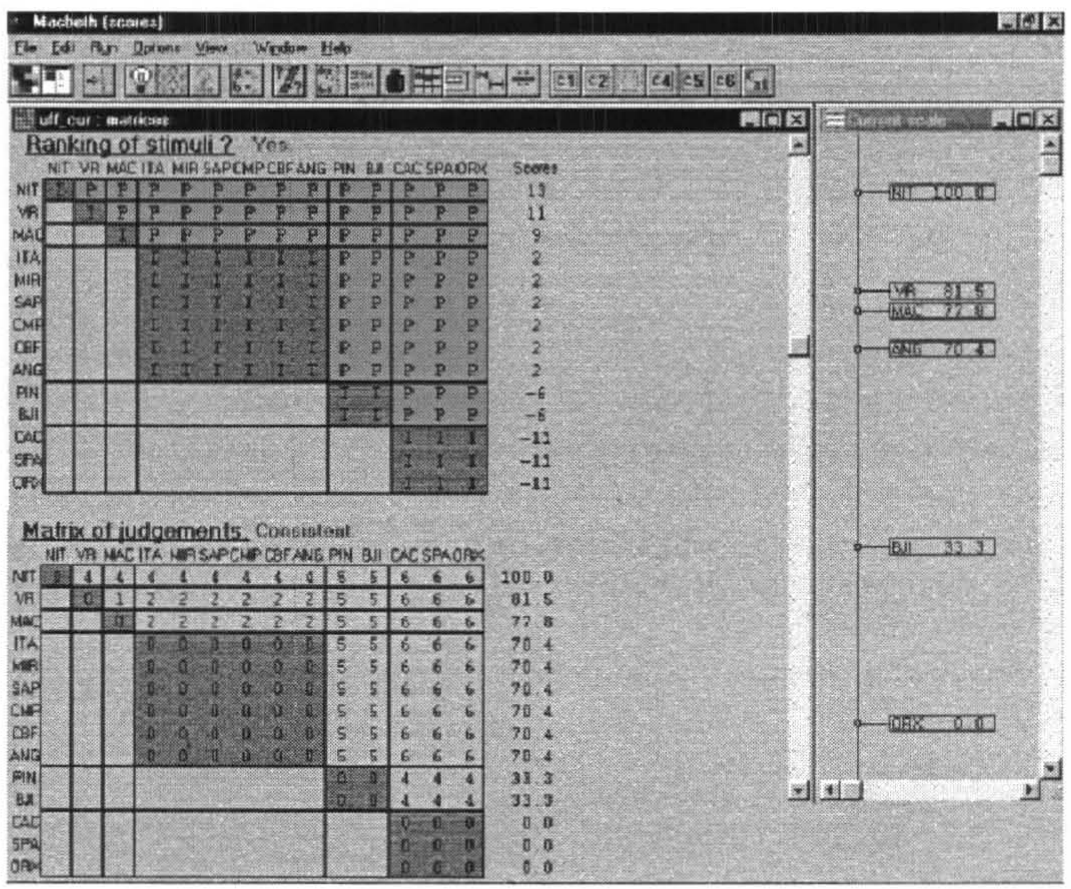

Figura 5: Tela do MACBETH para análise das alternativas no critério Tipo de Curso.

Tabela 1: Notas das alternativas nos critérios considerados.

\begin{tabular}{|c|c|c|c|c|c|}
\hline Município & $\begin{array}{c}\text { Tĩpo } \\
\text { de } \\
\text { curso }\end{array}$ & $\begin{array}{c}\text { Regularida } \\
\text { de do acesso }\end{array}$ & $\begin{array}{c}\text { Ações junto } \\
\text { à } \\
\text { comunidade }\end{array}$ & Instalações & $\begin{array}{c}\text { Corpo } \\
\text { docente }\end{array}$ \\
\hline Niterói & 100,00 & 100,00 & 100,00 & 100,00 & 100,00 \\
\hline Volta Redonda & 79,30 & 100,00 & 45,30 & 70,70 & 100,00 \\
\hline Campos dos Goytacazes & 70,40 & 100,00 & 62,60 & 70,70 & 100,00 \\
\hline Santo Antônio de Pádua & 70,40 & 96,70 & 45,30 & 29,40 & 96,80 \\
\hline Pinheiral & 29,70 & 96,70 & 13,10 & 70,70 & 100,00 \\
\hline Bom Jesus do & 29,70 & 96,70 & 13,10 & 70,70 & 100,00 \\
\hline Itabapoana & 70,40 & 80,30 & 13,10 & 0,00 & 43,80 \\
\hline Angra dos Reis & 76,00 & 77,00 & 0,00 & 0,00 & 56,40 \\
\hline Macaé & 70,40 & 70,40 & 13,10 & 0,00 & 56,40 \\
\hline Itaperuna & 70,40 & 73,80 & 0,00 & 0,00 & 43,80 \\
\hline Cabo Frio & 70,40 & 29,70 & 0,00 & 0,00 & 43,80 \\
\hline Miracema & 0,00 & 0,00 & 82,60 & 55,90 & 6,30 \\
\hline Oriximiná & 0,00 & 0,00 & 7,80 & 41,50 & 0,00 \\
\hline Iguaba Grande & 0,00 & 0,00 & 7,70 & 41,50 & 0,00 \\
\hline Cachoeiras de Macacu & & & & \\
\hline
\end{tabular}


município são residentes nas vizinhanças, se percorrem grandes distâncias no deslocamento ou, ainda, se recebem auxílio para hospedagem.

Neste último critério não foram feitas considerações sobre a "qualidade" do corpo docente. Em primeiro lugar, em municípios onde os professores comparecem apenas nos horários de aula, não estando disponíveis para outras atividades, é mais importante aumentar sua presença do que exigir uma eventual "qualidade". Por outro lado, o corpo docente em alguns municípios não é fixo, o que inviabiliza uma análise de sua "qualidade". Finalmente, "qualidade" docente é um assunto extremamente controverso, muitas vezes confundindo-se "qualidade" com titulação.

\section{JULGAMENTOS DE VALOR}

\section{- 5.1 Julgamento das Alternativas}

- 5.1.1 Critério Tipo de Curso

Dentre todas as alternativas, Niterói apresenta a maior nota por possuir um grande número de cursos em diversas áreas. Em seguida, apresenta-se Volta Redonda com três cursos de nível superior, Macaé com dois, Angra dos Reis, Cabo Frio, Campos dos Goytacazes, Itaperuna, Miracema e Santo Antônio de Pádua com um curso de nível superior cada. Em Pinheiral e Bom Jesus do Itabapoana existem cursos técnicos. Em Cachoeiras de Macacu, Iguaba Grande e Oriximiná não funcionam cursos. Na Figura 5 apresenta-se a tela do MACBETH com os julgamentos de valor e a escala cardinal (notas). A Tabela 1 resume as notas de todas as alternativas (municípios) nos cinco critérios considerados.

\subsubsection{Critério Regularidade do Acesso}

As cidades de Niterói, Volta Redonda e Campos dos Goytacazes têm tido, nos últimos anos, ingresso semestral regular, obtendo assim nota máxima neste critério. Santo Antônio de Pádua vem realizando vestibular anualmente, embora, em alguns anos, com vagas apenas para uma entrada semestral. Foi considerado equivalente a Pinheiral e Bom Jesus do Itabapoana que têm ingresso em todos os anos mas, por serem cursos técnicos, não possuem ingresso semestral. Em Angra dos Reis e Macaé tem havido ingresso em todos os anos, normalmente para apenas um semestre.

Esporadicamente ambos os municípios fazem ingresso para os dois semestres, o que acontece com maior freqüência em Angra dos Reis. Cabo Frio, Itaperuna e Miracema realizam vestibular para apenas um semestre. No entanto, desde a implantação dos cursos nestas cidades, em Cabo Frio nunca deixou de haver vestibular, o mesmo não ocorrendo em Itaperuna (um ano de falha) e em Miracema (maior número de anos sem vestibular do que com vestibular). Nos demais municípios, por não haver curso, não há ingresso.

\subsection{3 - Critério Atendimento junto à Comunidade}

A universidade possui um hospital universitário no município de Niterói (servindo como pronto-socorro municipal), onde possui ainda cinema, teatro, orquestra sinfônica, cursos de extensão, ações de atendimento comunitário e outros tipos de atividades. Estes fatos justificam nota máxima neste critério.

Em Oriximiná as atividades da UFF resumem-se ao atendimento à comunidade nas áreas social e de saúde. A UFF em Campos dos Goytacazes oferece alguns cursos de pós-graduação latu sensu, bem como convênios de prestação de serviços na área social. Em Volta Redonda e Santo Antônio de Pádua são realizados vários cursos de extensão, convênios com empresas elou eventos especiais (Semana da Matemática, por exemplo). Nos municípios de Pinheiral, Bom Jesus do Itabapoana, Angra dos Reis e Itaperuna o atendimento à comunidade é bastante reduzido, limitando-se a ações de venda da produção dos colégios agrícolas, eventuais cursos de extensão e atuação de Empresa Júnior. Em Iguaba Grande e Cachoeiras de Macacu existem alguns serviços prestados pelas fazendas, sendo que no primeiro há ainda uma colônia de férias. Nos demais municípios a ação comunitária é desprezível.

\subsubsection{Critério Instalações}

Em Niterói, a UFF conta com várias instalações próprias, acadêmicas e administrativas, distribuídas em vários locais na cidade, onde se realizam os diversos tipos de atividades desta instituição, justificando a nota máxima neste critério. Em Volta Redonda, Campos dos Goytacazes, Pinheiral e Bom Jesus do Itabapoana existem amplas instalações 


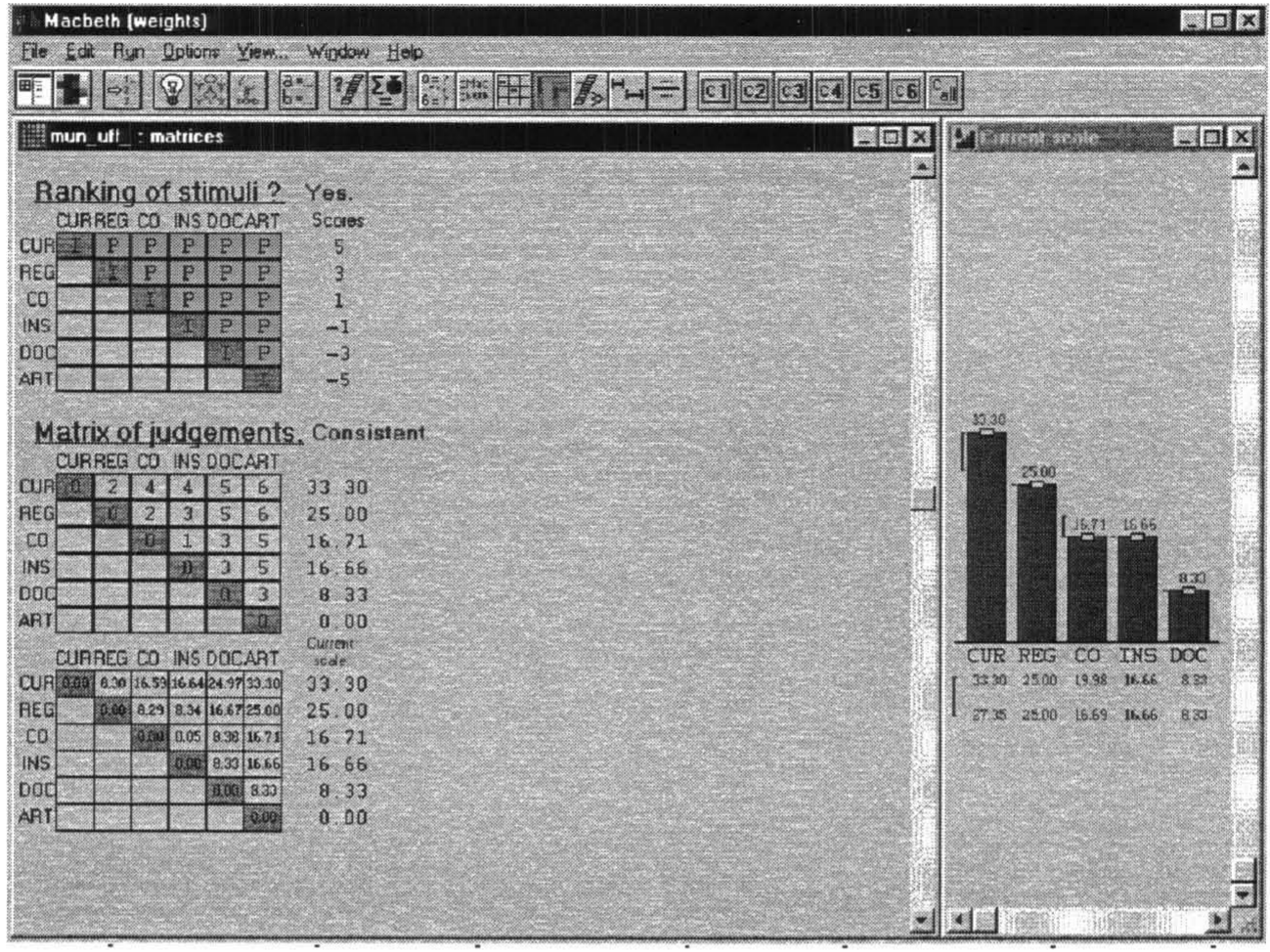

Figura 6: Tela do MACBETH para os pesos dos critérios.

Tabela 2: Pesos para os critérios.

\begin{tabular}{|c|c|}
\hline Critérios & Pesos \\
\hline Tipo de curso & 33,30 \\
\hline Regularidade do acesso & 25,00 \\
\hline $\begin{array}{c}\text { Ações junto à } \\
\text { comunidade }\end{array}$ & 16,71 \\
\hline Instalações & 16,66 \\
\hline Corpo docente & 8,33 \\
\hline
\end{tabular}


Tabela 3: Resultados finais indicativos da presença/visibilidade da UFF nos municípios.

\begin{tabular}{|c|c|}
\hline Município & Nota final \\
\hline Niterói & 100,00 \\
\hline Volta Redonda & 79,09 \\
Campos & 79,01 \\
Santo Antônio de Pádua & 68,15 \\
\hline Pinheiral & 56,36 \\
Bom Jesus do Itabapoana & 56,36 \\
Angra dos Reis & 49,36 \\
Macaé & 49,26 \\
Itaperuna & 47,93 \\
Cabo Frio & 45,54 \\
\hline Miracema & 34,52 \\
Oriximiná & 23,64 \\
\hline Iguaba Grande & 8,22 \\
Cachoeiras de Macacu & 8,20 \\
\hline
\end{tabular}

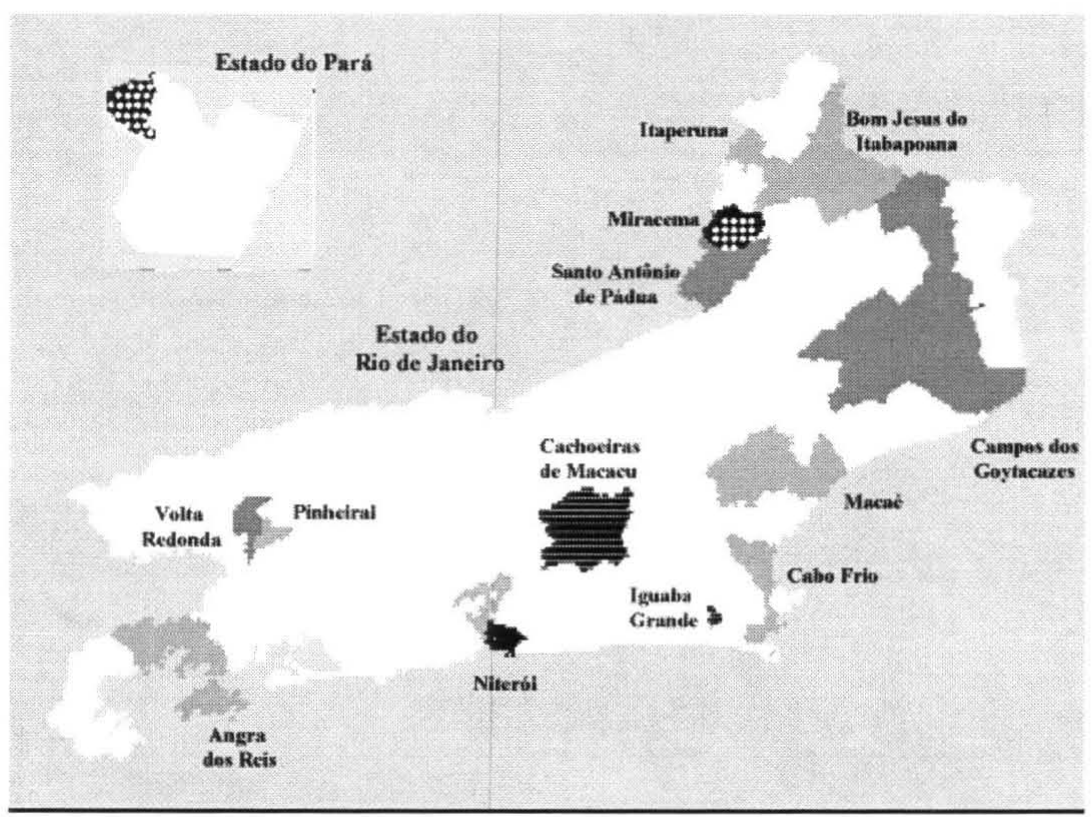

Figura 7: Divisão dos municípios em classes, de acordo com a visibilidade da UFF em cada município. 
próprias, com finalidades acadêmicas e administrativas, mas limitadas a um único local na cidade. Em Oriximiná a UFF possui instalações próprias somente destinadas ao atendimento à comunidade, sem utilização acadêmica (salas de aula, laboratórios, etc.). Iguaba Grande e Cachoeiras de Macacu contam apenas com fazendas $e$ suas respectivas sedes.

Nos demais municípios, a UFF usa colégios públicos el ou particulares com compartilhamento de atividades $e$ de espaços. Nestes municípios não é raro ocorrer troca do local cedido para as atividade da UFF. Deve-se ressaltar que em Santo Antônio de Pádua já existe um terreno, de propriedade da UFF, para construção das futuras instalações próprias.

\section{- 5.1.5 Critério Corpo Docente}

Niterói, Volta Redonda, Campos dos Goytacazes, Pinheiral e Bom Jesus do Itabapoana possuem corpo docente próprio e estável. Em Santo Antônio de Pádua existe uma situação semelhante, ressaltando-se, no entanto, que o corpo docente é reduzido e constituído, em grande parte, por professores visitantes que residem na Região Metropolitana do Rio de Janeiro, deslocandose apenas para ministrar suas aulas. Em Angra dos Reis, Cabo Frio, Macaé, Itaperuna e Miracema o quadro docente é constituído por professores lotados em Niterói que se deslocam apenas no dia de suas aulas. No entanto, em Macaé e Itaperuna já existe um pequeno número de professores visitantes lotados no local. Em Oriximiná não há corpo docente regular, sendo um pequeno número de professores designado por tempo determinado para assegurar as tarefas administrativas $e$ de orientação das atividades. Iguaba Grande $e$ Cachoeiras de Macacu não contam com corpo docente.

\subsection{Julgamento dos critérios}

Considerando-se que está sendo analisada uma instituição de nível superior, consideraram-se como mais atrativos os municípios onde funcionam cursos de nível superior, de preferência com regularidade de acesso. Como aspectos secundários, ações junto à comunidade e existência de instalações próprias são também fatores de visibilidade da instituição, com ligeiro predomínio do primeiro destes critérios. Finalmente, o tipo de corpo docente, embora seja um medidor de estabilidade $e$ presença da instituição, é pouco percebido pelo público em geral. Estas considerações, convenientemente introduzidas no programa MACBETH, fornecem como resultado a estrutura de pesos apresentada na Figura 6, resumida na Tabela 2 .

\section{RESULTADOS}

De imediato, nota-se na Tabela 1 que, ao contrário do usual em problemas multicritério, há uma alternativa (Niterói) que domina todas as outras. Verifica-se assim, que o município Niterói, por ter nota máxima em todos os critérios, é aquele com mais forte presença da UFF, quaisquer que sejam os pesos dos critérios.

A Tabela 3 mostra os resultados encontrados, ponderando-se as notas das alternativas em cada critério com os pesos designados aos mesmos.

Para uma melhor compreensão dos resultados, os municípios foram agrupados em 5 classes, propostas na Tabela 3 e visualizadas na Figura 7. O critério para a divisão em classes foi o de maior diferença absoluta entre os municípios extremos de cada classe, evitando-se que municípios com pontuação semelhante sejam alocados a classes diferentes.

\section{CONCLUSÕES}

$O$ método MACBETH permitiu que informações essencialmente qualitativas fossem transformadas em dados quantitativos que, quando agregados em uma função de utilidade aditiva, permitiram estabelecer uma hierarquização das alternativas em análise.

A análise multicritério é altamente sensivel a mudanças nos julgamentos de valor por parte dos decisores, o que pode provocar alterações na hierarquia. A divisão dos municípios em classes traz a vantagem de minimizar este efeito, por não ser analisada diretamente a posição do município, mas sim a que classe pertence.

As cinco classes usadas admitem várias interpretações. A primeira classe contempla apenas a sede da UFF, que se apresentou como alternativa dominante. A classe seguinte inclui três municípios (Santo Antônio de Pádua, Volta Redonda e Campos dos Goytacazes) onde a UFF tem atividades de graduação solidamente estabelecidas. Já na terceira classe encontram-se dois tipos de municípios: aqueles com atividades acadêmicas de nível médio solidamente estabelecidas e aqueles com 
cursos de nivel superior aparentemente bem estabelecidos, mas altamente dependentes de convênios com prefeituras. A quarta classe agrega um município fora do Estado do Rio de Janeiro, com atividades de assistência (normalmente pouco valorizadas), com um município que poderia estar na classe anterior, não fosse a extrema irregularidade de suas atividades. Finalmente, a última classe é composta pelos municípios onde a UFF tem pouco mais que a propriedade de parcas instalações.

Os resultados da quantificação da presença da UFF podem ser usados para o planejamento de futuras ações de interiorização desta instituição. Podem ainda ser usados para o planejamento do concurso Vestibular, já que indicam regiões onde, pelo fato de existir maior visibilidade, é lícito esperar maior aumento do número de candidatos.

Embora os resultados para unidades espacialmente organizadas sejam de fácil visualização e entendimento sob a forma de mapas (Soares de Mello et al., 2001), no presente artigo, devido ao reduzido número de alternativas e à impossibilidade técnica do uso de cores, optou-se pela divulgação na forma tabular e em mapas (estes em menor número), formas estas que se completam.

\section{REFERÊNCIAS BIBLIOGRÁFICAS}

\section{BANA E COSTA, C.A.; VANSNICK, J.C. Thoughts a} theoretical framework for measuring attractiveness by categorical based evaluation technique (MACBETH). In: Clímaco, J. (ed), Multicriteria Analysis, Springer-Verlag, Berlin, 1997.

BANA E COSTA, C.A.; VANSNICK, J.C. Uma nova abordagem ao problema da construção de uma função de valor cardinal: MACBETH. Investigação Operacional, $v$. 15, p. 15-35, 1995.

BANA E COSTA, C.A.; VANSNICK, J.C. MACBETH - an interactive path towards the construction of cardinal value functions. International Transactions in Operational Research, 1, p. 489-500, 1994.

BARBA-ROMERO, S.; POMEROL, J.C. Decisiones Multicriterio: Fundamentos Teóricos e Utilización Práctica, Colección de Economia, Universidad de Alcalá, 1997.

BOUYSSOU, D. Décision multicritèrie ou aide multicritèrie? Bulletin du Groupe de Travail Européen "Aide Multicritèrie à la Décision", series 2, n. 2, Printemps93, 1993.

CORRÊA, E.C. Construção de um Modelo Multicritério de Apoio ao Processo Decisório. Santa Catarina, EPS/UFSC, 1996. (Tese-Mestrado em Engenharia de Produção).

COSEAC. A UFF no estado do Rio de Janeiro, 2001. [Disponivel em http://www.coseac.uff.br/cidades/ cidades.htm]

DOIGNON, J.P. Threshold representations of multiple semiorders. SIAM Journal of Algebraic Discrete Methods, $v$. 8, p. 77-84, 1984.

GOMES, E.G.; LINS, M.P.E.; SOARES DE MELLO, J.C.C.B. Seleç̧ão do melhor município: integração $S I G$ -

Multicritério. Investigação Operacional, v. 22, n. 1, 2002.

ROY, B.; BOUYSSOU, D. Aide multicritèrie à la décision: méthods et cas. Economica, Paris, 1993.

SAATY, T.L. The Analytic Hierarchy Process. McGraw-Hill, New York, 1980.

SOARES DE MELLO, J.C.C.B.; GOMES, E.G.; LINS, M.P.E.; VIEIRA, L.A.M. Um caso de estudo da integração SIGDEA-MCDA: a influência de uma instituição de ensino superior em vários municípios do estado do Rio de Janeiro. Investigação Operacional, v. 21, n. 2, 2001. 